\title{
Lateral masking: Limitations of the feature interaction account
}

\author{
ANKE HUCKAUF and DIETER HELLER \\ Technical University of Aachen, Aachen, Germany \\ and \\ TATJANA A. NAZIR \\ C.N.R.S., Marseille, France
}

\begin{abstract}
Recognition performance for a target letter embedded in a string of characters is worse than that for targets presented in isolation. This lateral masking (LM) effect is known to depend on target eccentricity and spacing between target and flankers (Bouma, 1970), indicating that LM arises in early visual processing due to interactions among visual features. The feature interaction account would predict that flankers consisting of similar features produce similar LM effects and that differences in LM produced by different types of flanker diminish with increasing target eccentricity and decreasing spacing. However, in a series of six experiments, this prediction was shown not to be true. Flankers that did not access a higher level code (e.g., pseudoletters or rotated letters) produced more LM than standard letter flankers. Moreover, effects of different flanker types were most pronounced for medium target eccentricities and medium spacings for which recognition performance scores ranged between $40 \%$ and $60 \%$.
\end{abstract}

It is well established that the recognizability of letters diminishes with increasing retinal eccentricity (Aubert \& Foerster, 1857). Due to the marked decrease in visual resolution, letter identification accuracy declines even a few letter positions from fixation. For a given retinal eccentricity, however, there is yet another factor that affects recognition: Performance in parafoveal and peripheral vision is reduced when the target letter is flanked by nearby stimuli. This phenomenon is referred to as lateral masking (LM; Bouma, 1970; Massaro \& Cohen, 1994; Townsend, Taylor, \& Brown, 1971; Wolford \& Chambers, 1983).

There are three well-established properties of LM: (1) LM is more pronounced in the retinal periphery than in the center of the visual field (e.g., Bouma, 1970; Loomis, 1978; Strasburger, Harvey, \& Rentschler, 1991), (2) LM decreases with increasing spacing between target and flanking characters (e.g., Bouma, 1970; Heller, Huckauf, \& Nazir, 1995; Wolford \& Chambers, 1983), and (3) LM increases as the similarity between target and flankers increases (Egeth \& Santee, 1981; Estes, 1982). Since these properties appear to be sensory in nature, it is generally acknowledged that LM occurs at early visual processing stages (e.g., Estes, 1972; Strasburger et al., 1991; Wolford, 1975).

The research reported in this article was supported by Grant He $1192 /$ 2-2, 2-3 from the Deutsche Forschungsgemeinschaft to the second and third authors. It is part of a the first author's doctoral dissertation. The authors thank Hermann Müller, Phil Allen, John Flowers, Lisa Fournier, and Lester Krueger for helpful comments on previous drafts of the manuscript. Correspondence should be addressed to A. Huckauf, Institute of Psychology, Technical University of Aachen, Jaegerstr. 17-19, D-52056 Aachen, Germany (e-mail: anke@mail.psycho.rwth-aachen.de).
One of the most cited accounts for LM is feature interaction (Estes, 1972; Massaro \& Cohen, 1994; McClelland \& Rumelhart, 1981; Wolford, 1975). This account assumes that letter recognition is a hierarchically organized process that involves at least two processing levels: a first level where visual features are analyzed (often referred to as the feature level), and a subsequent level where letter identity is elaborated (the letter level). The visual input activates feature detectors, and the feature detectors all activate compatible letter nodes in a subsequent level (e.g., McClelland \& Rumelhart, 1981; Paap, Newsome, McDonald, \& Schvanefeldt, 1982; Sanocki, 1987, 1988). The term feature interaction means that features of adjacent letters compete with features of the target letter in activating a set of compatible letter candidates. The feature interaction account can provide an explanation of all three properties of LM: Given that the more similar the characters are, the more features they will have in common, similar characters increase the interaction among the features (see also Duncan \& Humphreys, 1992). Increasing the spacing between the characters should decrease the interaction between adjacent features. Increasing the eccentricity of the characters would reduce the acuity for feature analyses, leading to more confusion at the feature level.

Some characteristics of LM seem, however, at variance with an explanation in terms of early visual processes only. Specifically, variables that are assumed to influence early processing of sensory information, such as presentation duration, abrupt onsets, or positional cues, do not affect LM: LM does occur with unlimited and limited viewing time (Townsend et al., 1971), with both onsettransient and offset-transient flanker presentation (Miller, 
1991, Experiment 6) and at both cued and uncued target locations (Miller, 1991, Experiment 3).

Additionally, there is some evidence demonstrating effects of higher level processes in letter recognition tasks. For example, letter search is slower when searching through inverted letters (Greenberg \& Krueger, 1983; Krueger, 1984). Spacing variations produce differential effects on recognition performance, depending on the requirements of the task (Mewhort, Marchetti, \& Campbell, 1982). Egeth and Santee (1981) found that identification was lower for same letters even if the flanker was of a different form (A.a). However, since, in these studies, stimuli were highly visible, effects of higher level factors are more apparent than in studies concerned with LM where presentation is characterized by short presentation duration, eccentricity, and clutter.

But even with peripheral presentation of stimuli, some data seem to be inconsistent with feature interactions. Estes, Allmeyer, and Reder (1976) showed that the end effects - that is, the typical recognition advantage for the first letter and the last letter of a letter string (effects that are classically attributed to reduced interaction because of the flanking space)--remain even when the flanking space is substituted by flanking symbols, such as $\$$ or $\#$. Hence, these flanking symbols did not affect performance in the same way as flanking letters. Additionally, there are differential end effects in a letter recognition experiment, depending on the types of flanker: Whereas flanking letters produce end effects, flanking digits do not (Mason, 1982; Styles \& Allport, 1986, Experiment 1). These studies indicate that at least end effects cannot be fully explained in terms of feature interactions. Rather, higher level processes seem also to be involved in LM.

Studies in which the inner letters of a string were studied also produced inconsistencies with an explanation of LM in terms of early visual processes. Massaro and Cohen (1994; see also Massaro \& Klitzke, 1979) reported that $\mathrm{LM}$ effects produced by three different letter flankers or by three flanking dollar signs (e.g., recognizing the $n$ in the string aenl vs. $\$ \$ n \$$ ) were equivalent in magnitude. However, the dollar signs should mask the target less than three different letters, if only because of their homogeneity (see Heller et al., 1995). Moreover, Prinzmetal (1992) reported that target recognition performance was better for letters flanked by number signs (\#) than for letters presented in isolation. Obviously, an assumption of LM in terms of feature interactions would predict the opposite result.

However, according to recent letter recognition models, interactions between adjacent letters might arise not only at the feature level but also at the letter level. With a target letter embedded in other letters, both processing levels (i.e., feature and letter level) provide potential sources of interference. But, when flanking a target letter with nonletter characters, only the stage of feature analyses is common to target and flankers. Thus, interactions between the characters should occur only during feature processing. One might therefore expect letter flankers to have a stronger effect on target recognition performance than nonletter flankers.

Alternatively, the reduced interaction effects that are observed with nonletter flankers might be the result of higher level processes: The nonletter flankers in the above studies all consisted of familiar signs and symbols (e.g., $\$$, \#, and digits). Like letters, these characters are therefore likely to be coded at processing levels higher than the feature level. Via top-down feedback, any higher level code could conceivably attenuate noise in lower processing stages by grouping the elements of a stimulus (e.g., McClelland \& Rumelhart, 1981). Hence, the weak interference observed when target and flankers belong to different but meaningful stimulus categories could originate from several sources.

\section{THE PRESENT STUDY}

The present study investigated the role of feature interactions and higher level processes in LM. In particular, it was designed to test the predictions derived by the feature interaction account. To study interaction effects arising at the feature level and at some higher levels of processing, respectively, feature level activation is kept constant and, at the same time, higher level attributes are varied. Since there is no generally accepted definition of letter features, various approaches were followed in the series of experiments.

By hypothesis, letters in different orientations should produce a similar amount of activation at the featural level. Therefore, in Experiment 1, recognition performance for target letters flanked by normally oriented letters (the letter E) was compared with performance when flankers were presented in a different orientation. Experiment 1 was replicated with a different flanking letter, the letter $\mathrm{R}$ (Experiment 2) and with target letters displayed in a different orientation (Experiment 3 ) to eliminate some possible interpretations of the effect of flanker orientation. If the effect of flanker orientation that was obtained in Experiments 1 and 2 was due to feature processing, the same effect of flanker orientation should be observable with pseudoletter flankers. This prediction was tested in Experiment 4. In Experiment 5, LM generated by letter-like pseudoletters was compared with LM produced by letters: Given that pseudoletters and letters consist of similar features, pseudoletter flankers should produce the same amount of feature interaction as letter flankers. Thus, any differences in LM between letter-like pseudoletters and letter flankers should be due to interactions during letter coding. In Experiment 6 , interaction effects produced by digits were compared with those of letters. Unlike the flanking pseudoletters of Experiment 5, the digit flankers in Experiment 6 were coded at a higher level. If higher level codes modulate interaction effects, differences between the pseudoletter and digit flanking conditions are expected.

As mentioned above, LM depends on factors of retinal eccentricity and spacing (Bouma, 1970; Loomis, 1978; R. J. 
Jacobs, 1979). Assuming that decreasing spacing and increasing eccentricity increase feature interactions (but see Van der Heijden, 1992, for an interpretation of increased spacing as facilitation of target selection), different flanking features should result in different LM effects when target eccentricity is small and spacing between characters is big. For big eccentricities and small spacings, however, differences in LM because of differential flanking features should become less obvious. Thus, effects of spacing and eccentricity might be regarded as a control for differential feature interactions produced by different flanking characters. The portion of the LM effect that is due to other than feature interactions should therefore be less affected by variations of spacing and eccentricity. Since Bouma (1970) showed that target eccentricity and spacing are interacting variables, both variables were additionally manipulated in each experiment.

\section{GENERAL METHOD}

\section{Stimuli}

All 26 letters of the alphabet served as targets. Width and height of the letters were $3 \times 4 \mathrm{~mm}$, corresponding to a visual angle of $0.38^{\circ} \times 0.51^{\circ}$ at a viewing distance of $45 \mathrm{~cm}$. In the flanked conditions, two flankers, one on each side of the target, were displayed. All characters were typed in uppercase using the Windows 3.11 Arial font. Pseudoletters were constructed within a matrix of $10 \times$ 12 pixels, which corresponds to the size of the letters.

The stimuli were displayed at $1.08 \mathrm{~cd} / \mathrm{m}^{2}$ intensity on a $45-\mathrm{cd} / \mathrm{m}^{2}$ intensity background, resulting in a Michelson contrast of 1.05. The 14-in. CRT screen (Philips 4CM4270) had a refresh rate of $60 \mathrm{~Hz}$ and a resolution of $600 \times 800$ pixels. The experiment was controlled by ERTS (Experimental Run Time System; Beringer, 1993) version 3.0, which ran on an IBM-compatible 486 PC. ERTS ensures the synchronization of exposure time and refresh rate.

\section{Procedure}

The retinal eccentricity of the target letter was $1^{\circ}, 4^{\circ}$, or $7^{\circ}$. The spacing between the target and flankers was $0.4^{\circ}$ (normal), $1^{\circ}$, or $2^{\circ}$ (measured from center to center of the stimuli). Stimuli were presented in either the right or the left visual field. As stated above, all
26 letters of the alphabet served as targets. Additionally, flankers were varied. Thus, there were five independent variables - eccentricity (3), spacing (3), visual field (2), target letter (26), anit type of flanker (2)-yielding a total of 936 trials per participant As a control, target letters were presented in isolation at all eccentr cities in both visual fields $(26 \times 3 \times 2$, for a total of 156 trials $)$. All trials were presented in random order within one block. The dependent measure was percentage of correct target identification responses.

The participants viewed the screen binocularly from a distance of $45 \mathrm{~cm}$. The participants' head position was fixed by a chinrest. The sequence of events on an example trial is depicted in Figure 1. The participants started a trial by pressing a key on the keyboard in front of them. Five hundred milliseconds later, a fixation cross appeared in the center of the screen for $1,000 \mathrm{msec}$. Subsequently, the target and flanker string was exposed for $80 \mathrm{msec}$, and the participants had to report the target letter by pressing the appropriate letter key on the keyboard. If the participants were uncertain about the target letter's identity, they were instructed to make a guess. After 52 trials, about every $3 \mathrm{~min}$, the word PAUSE was displayed on the monitor, encouraging the participants to take a short break. They could continue the experiment by pressing the space key. An experimental session lasted about $2 \mathrm{~h}$.

Each participant was familiarized with the task in an initial practice session that lasted 20 min on average. Practice began with the presentation of all the letters that could occur as targets. Then, the participants were instructed to report the central letter of the string or, in case of isolated target presentation, the letter that was displayed. After that, they were shown six example strings, with unlimited viewing time, to demonstrate the central target position of the string and explain the task. Subsequently, there was a block of at least 50 practice trials, with $80 \mathrm{msec}$ of exposure time. During practice, every letter occurred at least once as target.

\section{EXPERIMENT 1}

The purpose of Experiment 1 was to investigate the effects of flankers that are comparable in terms of their visual features but that differ in terms of their ability to access an abstract identity code. Therefore, rotated letters were used as flankers and contrasted with normally oriented letter flankers. According to most assumptions about critical letter features (e.g., Bouma, 1978; A. M. Jacobs,
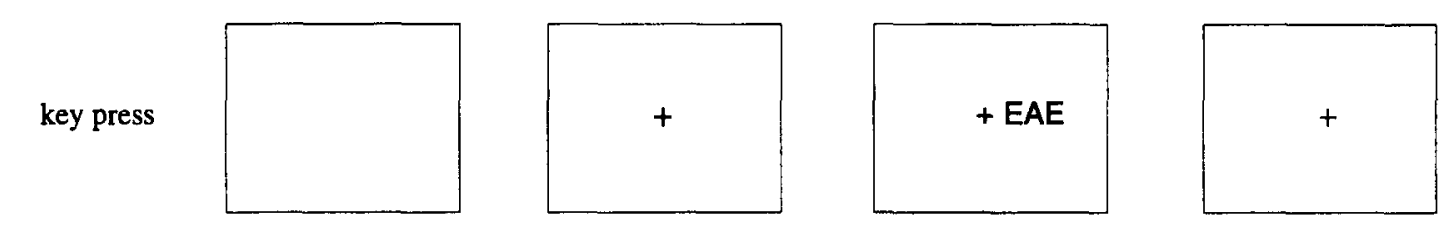

answer

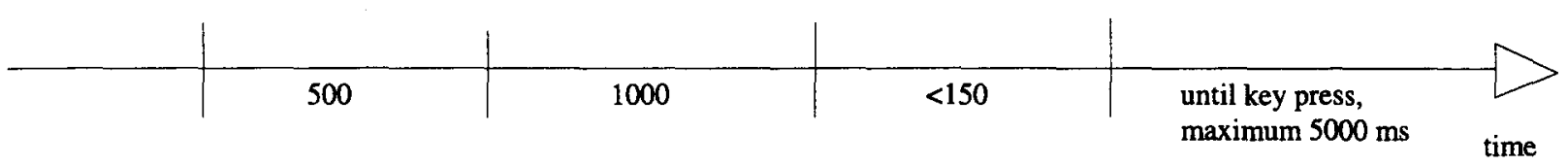

(ms)

Figure 1. Events in one trial. For 1,000 msec, a fixation cross appeared in the center of the monitor. Then, the stimuli were presented, and the participants' task was to identify the target letter and press the appropriate key on the keyboard. Five hundred milliseconds later, the next trial was started. 
Nazir, \& Heller, 1989; McClelland \& Rumelhart, 1981; Sanocki, 1987,1988 ), rotating a letter about $180^{\circ}$ in the picture plane leaves the letter features unchanged.

Normal upright letter flankers should produce interaction effects at every level of letter processing. With a $180^{\circ}$ rotation of the letter in the picture plane, critical features were assumed to stay the same. Hence, the amount of LM due to feature interactions should be constant for upright and rotated letters. However, for rotated letters, the coding of letter identity is known to take longer and to be prone to errors (Cooper \& Shepard, 1973). If access to an abstract letter identity code influences lateral masking, recognition performance should differ between targets flanked by rotated letters and targets flanked by upright letters.

\footnotetext{
Method

The letter $\mathrm{E}$ was selected as the flanking letter. Compared with an unrotated $E$, only the position of the vertical line segment of the character is changed when the $E$ is presented upside down. Moreover, when both flankers are identical, the overall visual similarity between the two flanking conditions is the same (i.e., in both conditions, the target is flanked by a vertical line on one side and three horizontal segments on the other side). Trial blocks consisted of 1,092 trials, including 156 trials with isolated letters and 468 trials per flanker condition. Five German graduate students, between 23 and 29 years of age, participated in Experiment 1. All participants reported normal or corrected-to-normal vision. The participants were paid or received course credit.
}

\section{Results}

Figure 2 shows the recognition scores as a function of eccentricity for the different spacing and flanking conditions. For reasons of clarity, scores were averaged across visual fields. Figure 2 shows the typical LM functionthat is, the decline in recognition accuracy with increasing eccentricity $[F(2,8)=605.47, p<.001]$ and with decreasing spacing $[F(2,8)=184.32, p<.001]$. Identification accuracy was consistently lower when flankers were presented upside down than when they were presented rightside up. Averaged across all conditions, the magnitude of flanker orientation effect was $4.95 \%[F(1,4)=15.29$, $p=.017]$. Other significant effects were the interactions of spacing $\times$ eccentricity $[F(4,16)=18.4, p<.001]$ and flanker $\times$ spacing $\times$ eccentricity $[F(4,16)=3.35, p=$ .036]. Both interactions result from larger accuracy differences between spacing conditions at the medium eccentricity of $4^{\circ}$. When target eccentricity was either $1^{\circ}$ or $7^{\circ}$, differences between the spacing and flanker conditions were less marked. The overall main effects did not change direction for any combination of the other variables.

\section{Discussion}

Contrary to our expectation, rotated letters produced greater LM than did upright letters. Assuming that LM occurs during feature processing, one would not expect to find any differences between the two conditions. As-

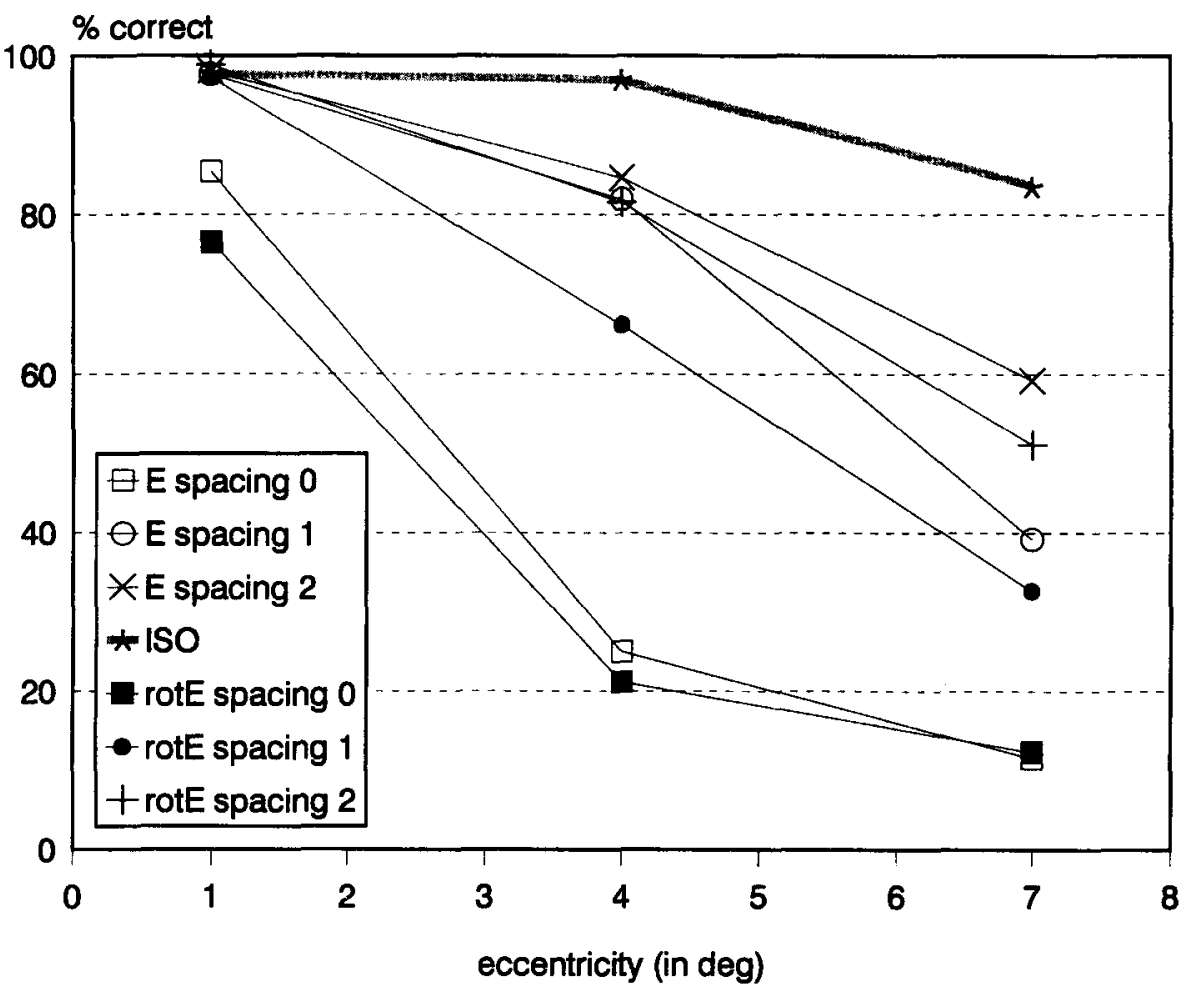

Figure 2. Target recognition accuracy for the three spacing conditions with upright and rotated $E$ flankers in Experiment 1 as a function of target eccentricity. The scores are averaged over participants and visual field. 
suming that $\mathrm{LM}$ involves interactions during feature coding and during letter coding, one would expect rotated letters to facilitate performance. Additionally, the effect of flanker orientation was obtained for every eccentricity and for every spacing condition. With foveal presentation, effects of category-based information are more apparent (e.g., Greenberg \& Krueger, 1983; Teichner \& Krebs, 1974); but, in the present experiment, presentation was characterized by eccentricity and clutter. If feature interactions were responsible for the effect of flanker orientation, one would have expected no difference between upright and rotated flankers, at least for large eccentricities and for small spacings. Since this outcome was not expected, Experiment 1 was replicated with a different flanking letter.

A significant interaction between target eccentricity and spacing was observed, replicating the results of Bouma (1970). Conceivably, this interaction was due to different levels of performance within each of the conditions. Ceiling-level performance at the target eccentricity of $1^{\circ}$ and floor-level performance at the target eccentricity of $7^{\circ}$ cannot be excluded as explanations for the effects. Since the main effect of eccentricity was manifest with all spacing conditions and the main effect of spacing was manifest with each target eccentricity, it seems plausible to maintain the assumption that the interaction between both variables was attributable to differing levels of performance.

\section{EXPERIMENT 2}

Since the outcome of Experiment 1 was unexpected, the experiment was replicated in Experiment 2 using a different flanking letter, the letter $\mathrm{R}$. $\mathrm{R}$ has the advantage that it (in contrast to the letter E previously used) consists not only of vertical lines but also diagonal and curved lines.

\section{Method}

For technical reasons, a different monitor, an EIZO 17-in. monitor with a refresh rate of $70 \mathrm{~Hz}$, was used. The resolution was $600 \times 800$ pixels, as before. The letters were $4 \times 5.6 \mathrm{~mm}$ in size, which corresponds to a retinal size of $0.51^{\circ} \times 0.71^{\circ}$ with the given viewing distance of $45 \mathrm{~cm}$. Eight participants, between 22 and 31 years of age, took part in the experiment.

\section{Results}

Figure 3 shows recognition performance as a function of eccentricity for the different spacing and flanking conditions. As in Experiment 1, the typical LM functions were obtained-that is, the decline in recognition performance with increasing target eccentricity $[F(1,7)=137.75, p<$ $.001]$ and decreasing spacing $[F(2,14)=228.43, p<$ $.001]$. Identification accuracy was consistently lower when flankers were presented upside down rather than right-side up. Averaged across all conditions, the effect of flanker orientation was $9.86 \%[F(1,7)=96.39, p<$ $.001]$. Replicating Experiment 1, other significant effects

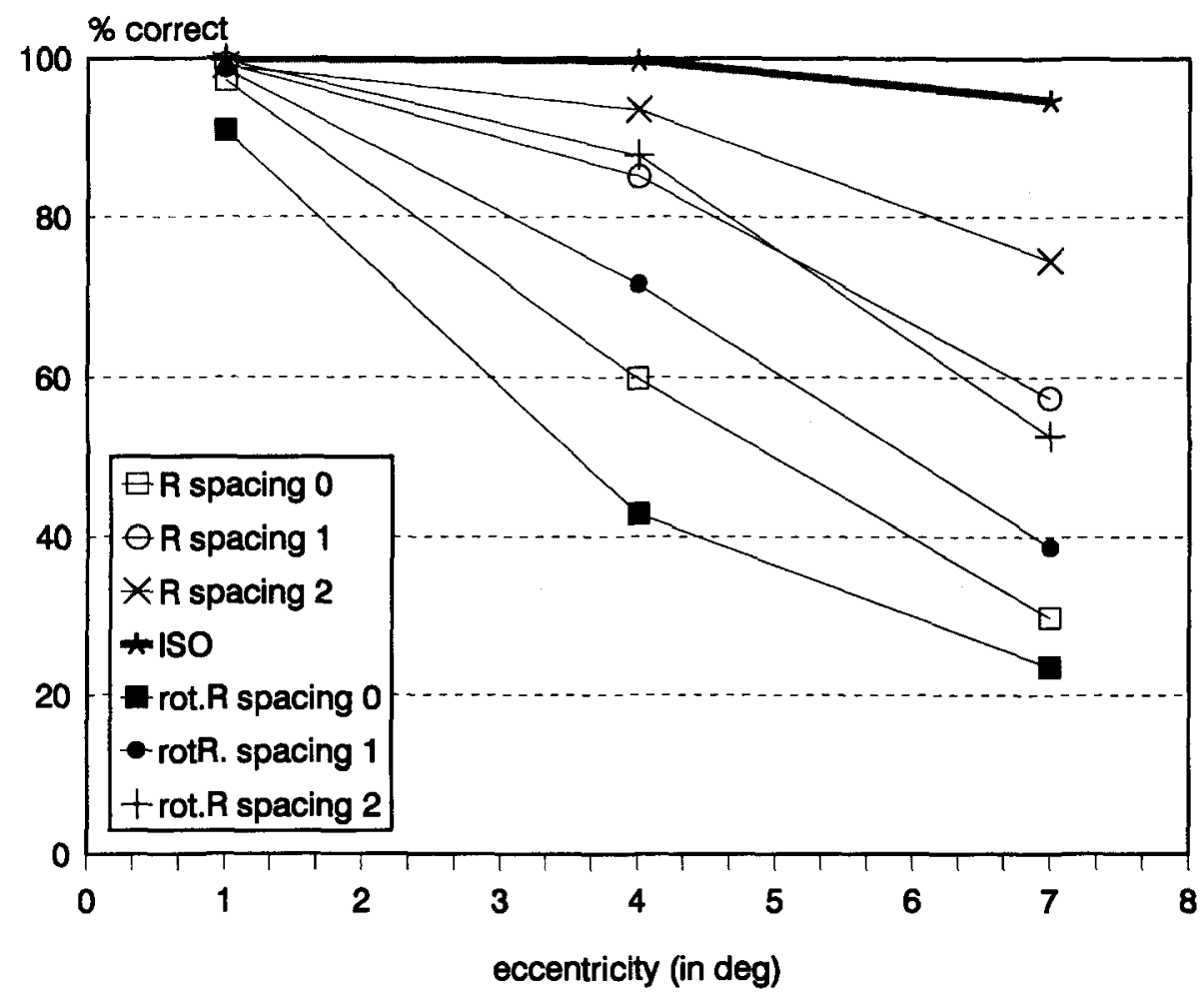

Figure 3. Target recognition performance depending on target eccentricity separately for the three spacing conditions and rotated and upright $R$ flankers in Experiment 2 . The scores are averaged over participants and visual field. 
were the interaction between target eccentricity $\times$ spacing $[F(4,28)=37.54, p<.001]$ and the interaction of eccentricity $\times$ spacing $\times$ flanker $[F(4,28)=6.12, p=.001]$.

\section{Discussion}

Experiment 2 completely replicated the results of Experiment 1 . In both experiments, rotated flanking letters produced stronger LM effects than did upright flankers. Differences between upright and rotated flanker presentation were observable for every eccentricity and every spacing condition. Before considering the significance of these results, different possible interpretations of this effect were ruled out. A post hoc analysis was conducted to study the effect of facilitated processing of the flankertarget-flanker strings that produced a meaningful word. With upright flankers, in a few trials, words were presented. Hence, one might argue that word processing was responsible for the better recognition performance when using upright flankers. Experiment 3 was conducted in order to test whether rotated flankers per se impair target recognition, using target letters that were also rotated. Experiment 4 was conducted in order to test the assumption that features of rotated and upright letters do not differ, using rotated pseudoletter flankers.

\section{Post Hoc Analyses: Word Advantage}

One possible explanation of the results of Experiments 1 and 2 would be that the superiority for target recognition with upright flankers might have been due to word processing: With upright flankers, a three-letter string was presented, thus allowing processing of higher level attributes of the complete string, whereas, with rotated flankers, target letter recognition could not benefit from word processing. To test this interpretation, a post hoc analysis was conducted, comparing recognition performance for letters in words, pseudowords, nonwords, and strings of same letters (EEE, RRR). For Experiment 1, the word group consisted of the strings EHE, EDE, and the familiar English word EYE. For Experiment 2, only one string (RAR) was counted as a word. The pseudoword category consisted of the strings EBE, ECE, EFE,
EGE, EIE, EJE, EKE, ELE, EME, ENE, EPE, ERE, ESE, ETE, EUE, EVE, EWE, EXE, and EZE for Experiment 1 and the strings RER, RIR, ROR, and RUR for Experiment 2. Except strings of identical letters (EEE, RRR), which were grouped separately, all other strings were included in the nonword category. If word processing was responsible for the effects, one should expect better performance for upright flankers when the string formed a word and no difference between upright and rotated flankers for nonword contexts. Table 1 gives the recognition performance for the different string categories.

Except for the pseudoword category in Experiment 1, for each context target recognition, accuracy was lower when flankers were presented upside down. This was even the case when the string consisted of three same letters (EEE, RRR). Interestingly, recognition performance for targets in strings of the same letters was worst in both experiments. This might indicate either a discriminability problem (Shapiro \& Krueger, 1983) or a tendency (response bias) to inhibit or suppress the reporting of the flanking letter. Further studies should be conducted to examine both interpretations. Going back to the question at issue, the effects of context cannot account for the orientation effects.

\section{EXPERIMENT 3}

A possible explanation for the different $L M$ effects produced by upright and rotated flankers might be that rotated letters produce special flanking effects that differ from those of upright flankers. Experiment 3 was conducted to rule out this possibility by using rotated target letters. If rotated flankers per se impair target recognition, rotated flankers should hamper target recognition when using rotated target letters.

\section{Method}

The same method as in Experiment 1 was applied. The only difference was the orientation of the target letters. In Experiment 3, target letters were all rotated $180^{\circ}$ in the picture plane, thus matching the orientation of the rotated flankers. Five participants, between 25 and 29 years of age, took part in Experiment 3.

Table 1

Percentage of Correct Target Recognition (\% Correct) and Number of Trials $(n$ ) for the Different Context Conditions and for Upright and Rotated Flanker Presentation in Experiments 1 and 2

\begin{tabular}{|c|c|c|c|c|c|c|c|c|c|c|}
\hline \multirow[b]{3}{*}{ Flanker } & \multicolumn{10}{|c|}{ Context } \\
\hline & \multicolumn{2}{|l|}{ Word } & \multicolumn{2}{|c|}{ Pseudoword } & \multicolumn{2}{|c|}{ Nonword } & \multicolumn{2}{|c|}{$\begin{array}{c}\text { Three Same } \\
\text { Letters }\end{array}$} & \multicolumn{2}{|c|}{ Total } \\
\hline & $\%$ Correct & $n$ & $\%$ Correct & $n$ & $\%$ Correct & $n$ & $\%$ Correct & $n$ & $\%$ Correct & $n$ \\
\hline \multicolumn{11}{|c|}{ Experiment 1} \\
\hline Upright & 68.89 & 270 & 65.91 & 1,710 & 60.74 & 270 & 42.00 & 90 & 64.74 & 2,340 \\
\hline Rotated & 60.00 & 270 & 66.41 & 1,710 & 56.30 & 270 & 39.00 & 90 & 59.79 & 2,340 \\
\hline \multicolumn{11}{|c|}{ Experiment 2} \\
\hline Upright & 93.06 & 144 & 75.87 & 576 & 76.98 & 2,880 & 64.00 & 144 & 76.95 & 3,744 \\
\hline Rotated & 62.50 & 144 & 67.01 & 576 & 68.09 & 2,880 & 58.00 & 144 & 67.09 & 3,744 \\
\hline
\end{tabular}




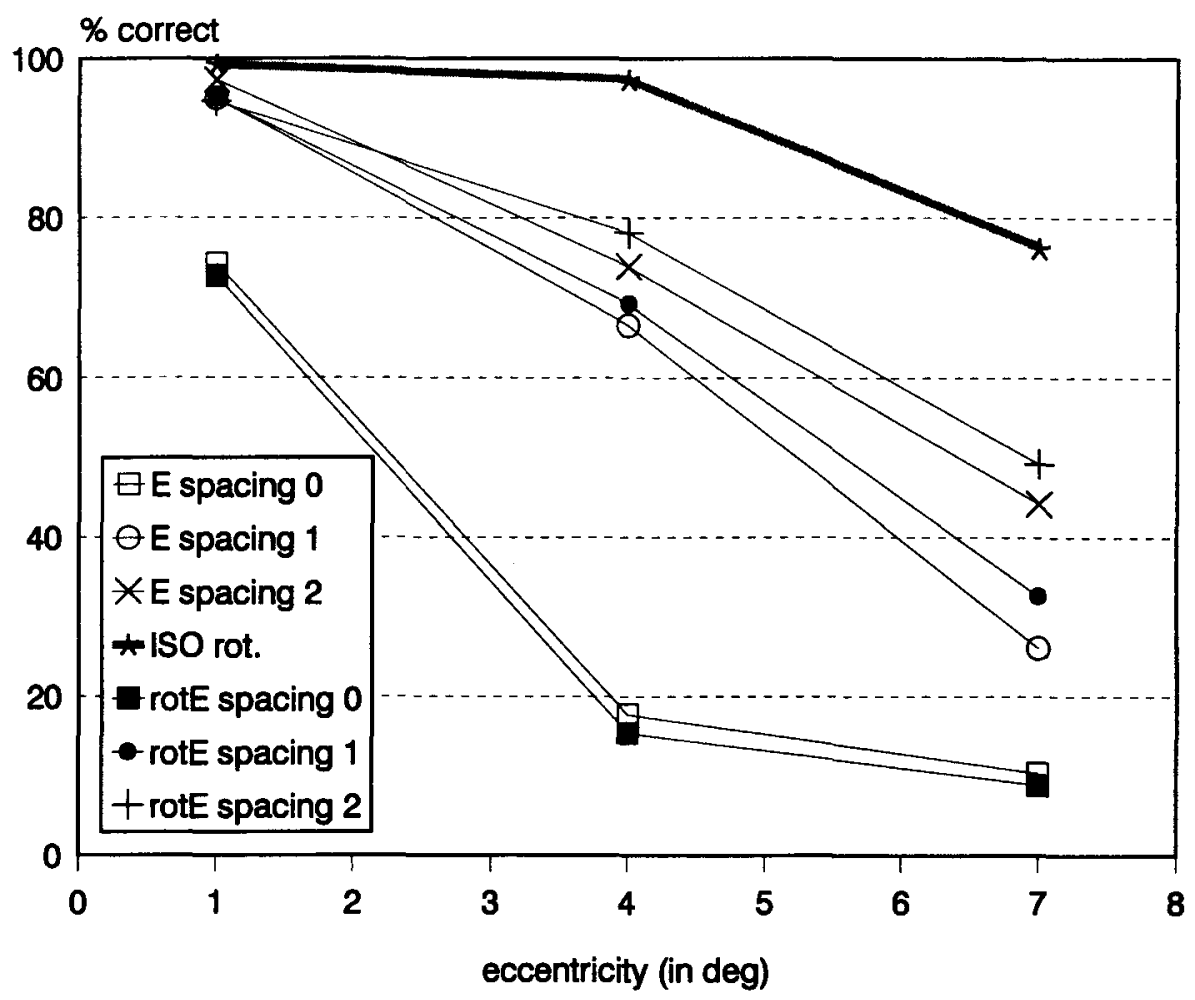

Figure 4. Recognition accuracy for rotated target letters as a function of target eccentricity for the three spacing conditions and upright and rotated $E$ flankers in Experiment 3 . The scores are averaged over participants and visual field.

\section{Results}

Figure 4 shows recognition performance for the three spacing conditions as a function of target eccentricity. Target recognition accuracy did not depend on flanker orientation $[0.28 \%$ better recognition performance when rotated targets were flanked by rotated letters; $F(1,4)<1]$. As in Experiment 1, there were significant main effects of target eccentricity $[F(2,8)=359.91, p<.001]$ and spacing between target and flankers $[F(2,8)=89.52, p<$ $.001]$, as well as a significant interaction between target eccentricity and spacing $[F(4,16)=18.28, p<.001]$.

\section{Discussion}

Contrary to Experiments 1 and 2, rotated flankers in Experiment 3 did not impair target recognition performance when compared with upright flankers. Thus, it can be concluded that rotated flankers per se do not impair target recognition performance. All other independent variables produced the expected effects (i.e., in Experiment 3 , the typical lateral masking functions were also obtained). Target recognition accuracy was lower with increasing eccentricity and with decreasing spacing, and there was a significant interaction between eccentricity and spacing. The difference between Experiment 3 and Experiments 1 and 2 was that target letters were also rotated. Since the effect of flanker orientation cannot be observed with rotated target letters but can be observed with upright target letters, the results of Experiment 3 argue that feature interactions alone cannot account for the LM effects. Instead, some higher level attributes of the flankers seem to be involved in LM.

\section{EXPERIMENT 4}

The results of Experiments 1 and 2 indicated that rotated flankers interfere more strongly with target recognition than do upright flankers. Given the assumption that rotating a letter $180^{\circ}$ in the picture plane does not change its visual letter features, these results question the notion of LM as an interaction among visual features. However, this assumption might be wrong. By rotating the flankers in Experiments 1 and 2, the position of the vertical line segment was changed from left to right (espe-

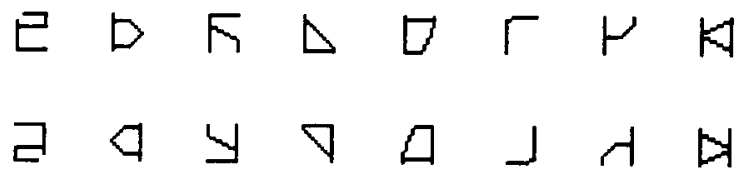

Figure 5. Eight pseudoletters that served as fankers in Experiments 4 and 5. In Experiment 4, in one condition, the pseudoletters were presented in an upright orientation (upper row); in the other condition, they were rotated $1 \mathbf{8 0}^{\circ}$ in the picture plane (lower row). 
cially for the flanker E). Perhaps this marked feature was responsible for the LM effects in Experiments 1 and 2.

The purpose of Experiment 4 was to investigate whether the relative position of the vertical line segment changes the LM effect. To rule out higher level processing, flankers were used that had no higher level representation. These pseudoletter flankers all consisted of a vertical line segment on their left-hand side (see Figure 5). If the orientation effects in Experiments 1 and 2 were due to a change of the relative position of the vertical line segment, one would expect the same orientation effect with the pseudoletter flankers. We opted for these symbols because they had been shown to be equivalent to letters in terms of speed of visual search (Kehrer, 1985). The advantage of using Kehrer's symbols in our experiments was their independently established visual similarity with real letters. Thus, we could safely proceed on the assumption that these characters were comparable to letters in terms of visual features, but they had no higher level representation.

\section{Method}

Eight characters from the study of Kehrer (1985) were used as flankers. The characters are shown in Figure 5. All stimuli had a vertical line on the left-hand side. As in Experiment 1, in one condition, upright items were presented as flankers; in the other condition, the same items were presented rotated $180^{\circ}$ in the picture plane.
Six participants, between 24 and 27 years of age, took part in the experiment.

\section{Results}

The recognition scores for the target letters as a function of target eccentricity are shown in Figure 6 . The mean difference between rotated and upright flankers was $0.4 \%$, which was not significant $[F(1,5)<1]$. Apart from the absence of a flanker orientation effect, the results were comparable to those of Experiment 1. In particular, the effects of eccentricity and spacing were also obtained with nonletter flankers [eccentricity, $F(2,10)=$ $321.49, p<.001$; spacing, $F(2,10)=180.18, p<.001$; eccentricity $\times$ spacing, $F(4,20)=3.61, p=.023$ ]. Again, the spacing effect was evident at each eccentricity, and the effect of target eccentricity was evident at each spacing condition. The interaction effects were due to larger spacing effects for the medium eccentricity of $4^{\circ}$ and smaller spacing effects when target eccentricity was either $1^{\circ}$ or $7^{\circ}$.

\section{Discussion}

With pseudoletter flankers, there was no differential LM effect when flankers were presented in different orientations. Thus, the relative position of the vertical line segment did not affect the magnitude of LM. This result

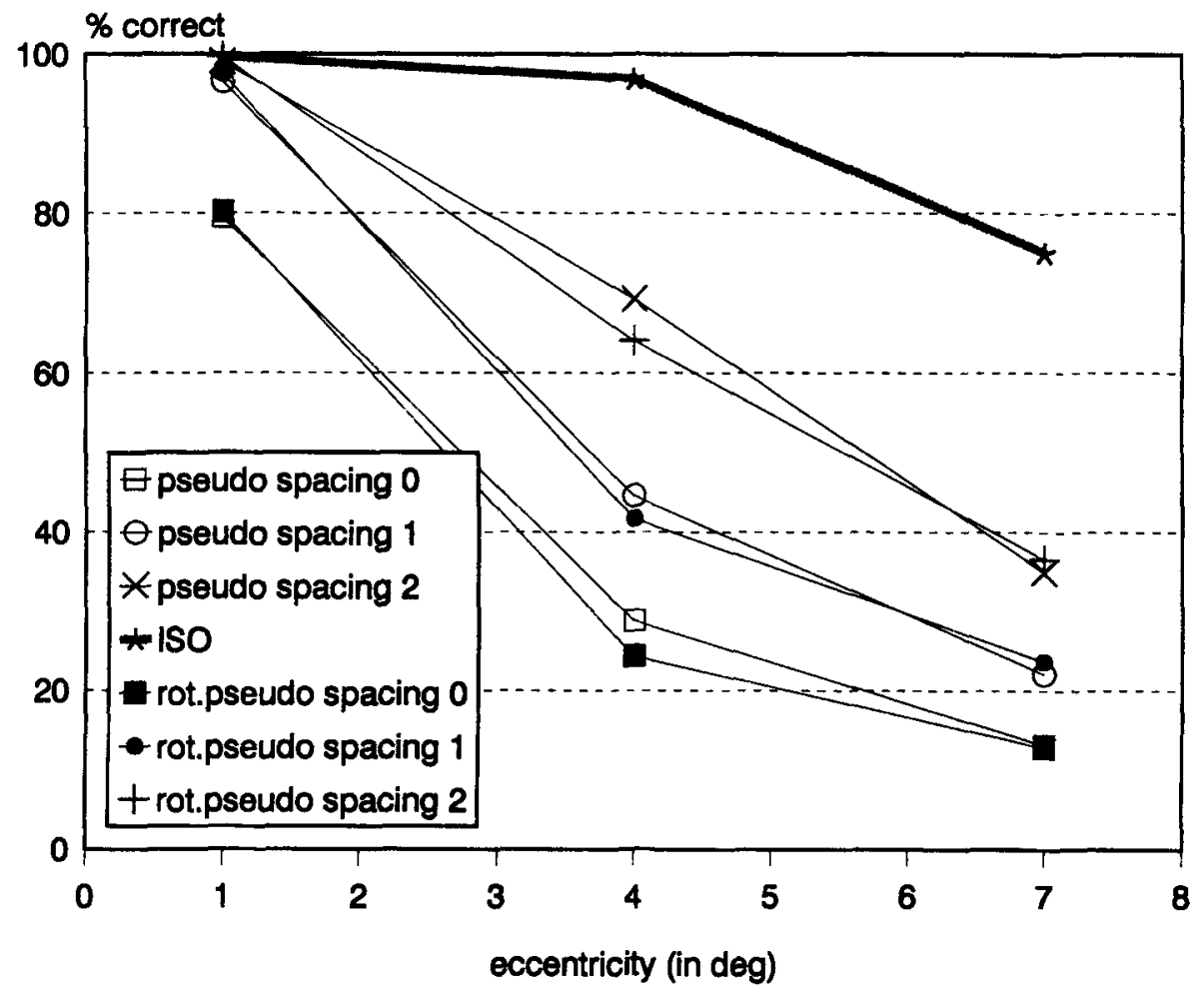

Figure 6. Target recognition performance depending on target eccentricity for the three spacing conditions and rotated and upright pseudoletter flankers in Experiment 4 . The scores are averaged over participants and visual field. 
has implications concerning the interpretation of Experiments 1 and 2. In Experiments 1 and 2, letter flankers in different orientations produced differential LM effects. Experiment 4 showed that pseudoletter flankers do not produce similar effects. Since pseudoletter flankers can interact with target recognition only at a featural level, this result argues that feature interactions cannot be responsible for the effect of flanker orientation.

Thus, an alternative explanation for the effect of flanker orientation is required. As pointed out above, rotated and upright letters do not differ in terms of relevant visual features. The difference between rotated and upright letter flankers on the one hand and rotated and upright pseudoletter flankers on the other seems to be the differential access to higher level representations. Moreover, the results of Experiments 1,2, and 4 favor the idea that access to an abstract letter code provided by flankers facilitates recognition of the target letter. In other words, target and flankers interact not only during feature processing but also during higher level processing. If flankers access an abstract letter code, this can decrease the interference from simultaneously presented flanking characters.

\section{EXPERIMENT 5}

The goal of Experiment 5 was was to investigate the effect of flanking characters that bring out the same amount of feature interaction but do not have access to a higher level representation. As suggested by the results of Experiments 1 and 2, flanking characters that do not access the letter level should produce more LM than letter flankers. Since it is difficult to design characters that satisfy all theoretical demands of feature construction, in Experiment 5, empirically derived letter-like symbols were used as comparison stimuli with letters. The letter-like flankers were the same eight pseudoletters presented in Experiment 4.

\section{Method}

The same experimental method as before was used. The flanking set consisted either of the eight pseudoletters presented in Figure 5 (upper row) or of the eight letters B, E, H, K, N, P, R, and U. Thus, all flanking characters were of the same size (width and height) and were of the same stroke width; each flanking character had a vertical line segment on the left-hand side. On each side of the target letter, two items either from the set of eight pseudoletters or from the set of eight letters served as flankers. For every trial, the flankers were chosen with the restriction that identical stimuli could never appear in one string. Six participants, between 25 and 33 years of age, were tested in the experiment.

\section{Results}

Both eccentricity and spacing produced the expected effects on recognition scores [eccentricity, $F(2,10)=$ $624.34, p<.001$; spacing, $F(2,10)=204.72, p<.001$; eccentricity $\times$ spacing, $F(4,20)=11.67, p<.001]$. Figure 7 shows the average recognition performance as a function of retinal eccentricity, separately for the various spacing and flanker conditions. Recognition accuracy for

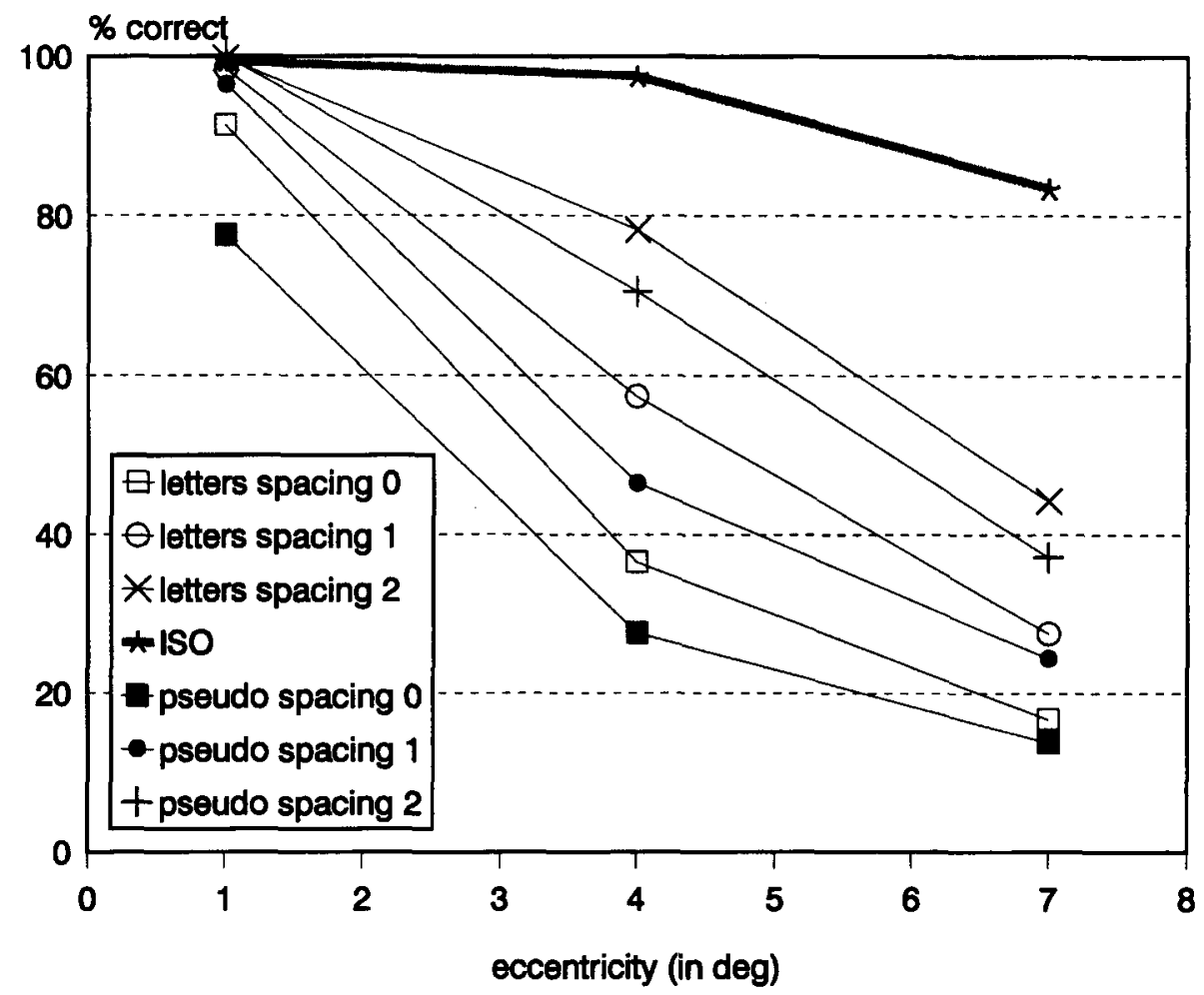

Figure 7. Target recognition accuracy depending on target eccentricity for the three spacing conditions and pseudoletter flankers versus letter flankers in Experiment 5. The scores are averaged over participants and visual field. 
targets flanked by pseudoletters was lower than for targets flanked by letters $[6.2 \% ; F(1,5)=71.98, p<.001]$. Other significant effects were the interaction of flanker type $\times$ eccentricity $[F(2,10)=7.83, p=.009]$ and the interaction of flanker type $\times$ eccentricity $\times$ spacing $[F(4,20)=5.55, p=.004]$. These interactions were due to the smaller differences between the flanking and spacing conditions for the target eccentricities of $1^{\circ}$ and $7^{\circ}$, relative to the medium target eccentricity of $4^{\circ}$.

\section{Discussion}

The main finding of Experiment 5 was that letter flankers reduce $L M$ effects, relative to pseudoletter flankers. The result that letter-like nonletter stimuli mask a target letter more effectively than do letter flankers is not always found in the literature. For example, Styles and Allport (1986, Experiment 3) studied the masking effects of letter-like forms. Letter-like forms masked a target letter less effectively than did letter flankers when the letter flankers were derived from the same response set as the target. However, when the letter flankers were from a response set different from the one of the target, LM produced by those letter flankers was the same as LM produced by letter-like forms. Hence, their results also show that $\mathrm{LM}$ is at least in part due to higher level processes. Additionally, they did not state their criteria to refer to their characters as letter-like. Since there is no generally accepted view of relevant letter features, independently established stimuli should be used as a comparison with letters.

The hypothesis that higher level attributes of the flanking characters are also processed was confirmed when using letter-like pseudoletters as flankers. Thus, target and flankers interact not only during feature processing but also during letter coding. Moreover, the data argue that letter coding works via reducing early interactions. Contrary to the predictions of the feature interaction account, there was no evidence in the data to suggest that the effect of flanker type decreases with increasing eccentricity and/or decreasing spacing.

\section{EXPERIMENT 6}

The experiments reported thus far show that nonletter flankers decrease target recognition performance more than letter flankers do. However, there ought to be a beneficial effect of flankers that do not belong to the letter category. If all items are processed and the interaction effects are due to the processing of higher level attributes (as was indicated by different strengths of the interaction effects, depending on the flanker type), there ought to be less interference when higher level attributes of target and flankers differ from each other. Of course, differences in higher level attributes, such as orientation or category of a character, can facilitate target recognition performance only when they are detected. One might therefore conclude that the rotated letter flankers and the pseudoletter flankers are not processed up to the point at which it is established that they belong to the letter category. If the flanking set consisted of nonletters that can be processed as fast and as accurately as letters, a facilitatory effect of those nonletter flankers ought to be found. To examine this hypothesis, flankers from a different set of characters might be used for comparison. Unfortunately, there are no characters that are as well known as letters and consist of the same visual features. Nevertheless, digits can be regarded as a set of stimuli that can be processed at least as easily and accurately as letters. Digits that flank a target letter should enhance recognition performance, relative to flanking letters. In Experiment 6, the masking effects produced by digits and letters were compared. The expectation was that it would be easier to recognize a letter flanked by digits than to recognize a letter flanked by other letters. Featural differences between letters and digits should manifest in increasing differences between digit and letter flankers with increasing eccentricity and decreasing spacing.

\section{Method}

All digits were used except for 1 and 0 , which are confusable with the letters $I$ and $O$. Eight letters that were visually similar to the digits were used as comparison stimuli: $Z$ (2), E (3), H (4), G (5), S (6), T (7), B (8), and $R(9)$. The same experimental procedure as before was used. Six participants, between 23 and 31 years of age, were tested in this experiment.

\section{Results}

Figure 8 shows the average recognition scores for the three spacing conditions and the two flanker conditions as a function of target eccentricity. As can be clearly seen, digit flankers produced superior target letter recognition scores, relative to letter flankers. The mean difference between the two conditions was $7.1 \%$, which is significant $[F(1,5)=17.87, p=.008]$. The typical lateral masking function was again obtained: decreasing recognition performance with increasing eccentricity $[F(2,10)=$ $190.65, p<.001]$, and decreasing spacing $[F(2,10)=$ $66.55, p<.001]$, as well as the interaction between both variables $[F(4,20)=13.60, p<.001]$. Other significant effects were the interactions of flanker type $\times$ eccentricity $[F(2,10)=5.58, p=.024]$ and flanker $\times$ spacing $[F(2,10)=5.50, p=.024]$. As in the previous experiments, interactions occurred because differences between spacing conditions and flanker conditions were most pronounced at the medium target eccentricity of $4^{\circ}$ and less pronounced when the target was displayed at $1^{\circ}$ or $7^{\circ}$ of eccentricity. A similar account applies to the interaction between flanker and spacing. The most pronounced difference between digit and letter flankers could be observed with a spacing of $1^{\circ}$, whereas this difference was least pronounced with the largest spacing of $2^{\circ}$.

\section{Discussion}

The results of Experiment 6 show that it is easier to recognize a letter flanked by digits than a letter flanked by other letters. Of course, it cannot be excluded that part of this effect was due to differences between visual fea- 


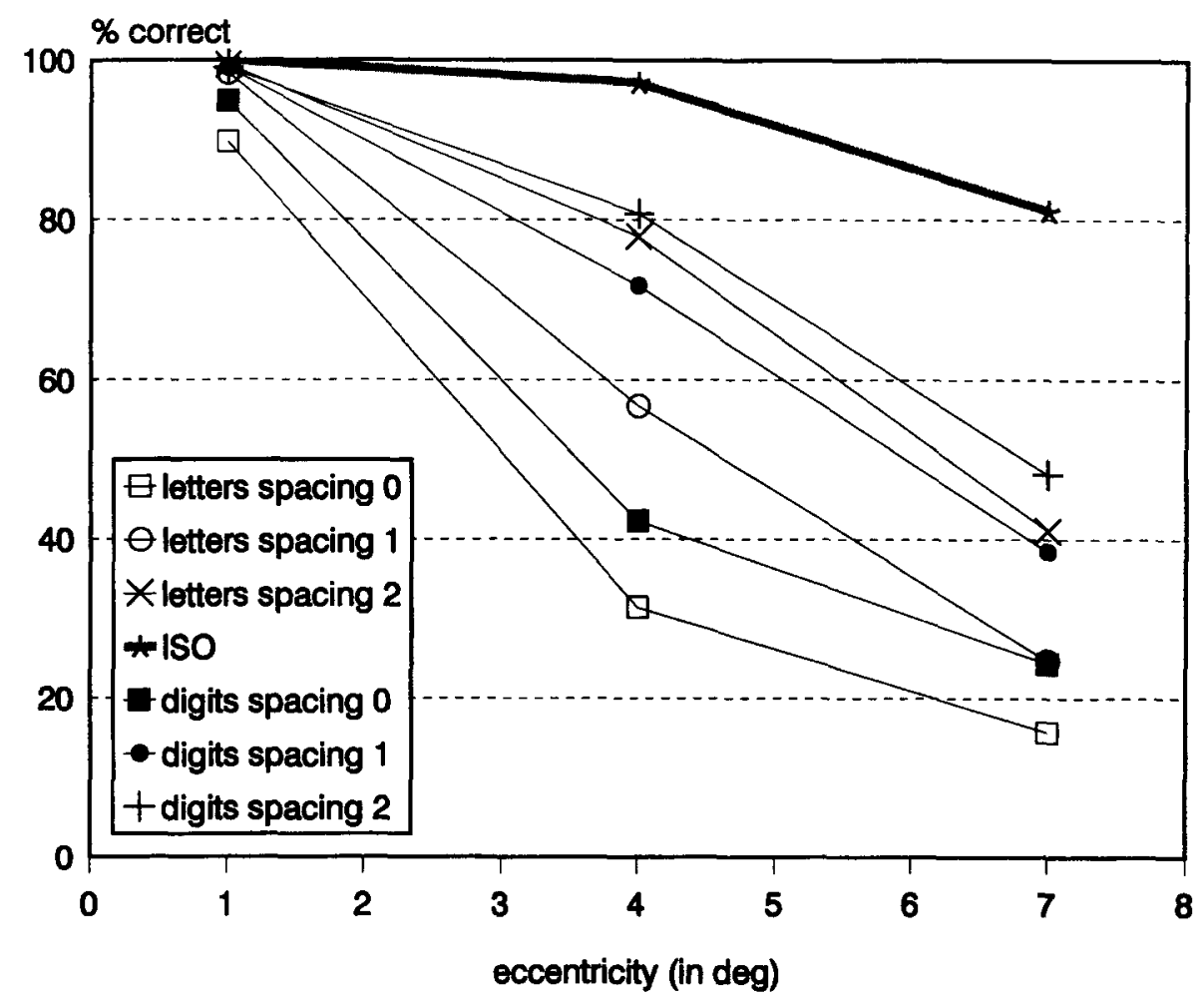

Figure 8. Target recognition performance as a function of target eccentricity for the three spacing conditions and digit versus letter flankers in Experiment 6. The scores are averaged over participants and visual field.

tures of letter and digit flankers (Krueger, 1984). However, if feature interactions are responsible for this effect, one has to explain why the difference between digit and letter flankers is most pronounced for the medium eccentricity and spacing conditions and occurs even when the target is displayed at $7^{\circ}$ of eccentricity with a spacing of $2^{\circ}$ between target and flankers. Restated, feature interactions alone cannot account for the effects obtained here. Considered in connection with the results of Experiments 1 and 5, the findings of Experiment 6 further support the notion that access to a higher level code reduces the flanker effects.

\section{GENERAL DISCUSSION}

The present study was designed to examine effects of flanker processing on target recognition. The very existence of LM indicates that irrelevant flanking letters are processed at least to some extent. The question at issue was to determine precisely which attributes of the flankers are processed, in order to clarify the role of low-level feature interactions and higher level coding processes in generating LM. On the basis of current models of letter and word recognition (e.g., McClelland \& Rumelhart, 1981; Sanocki, 1987, 1988), it was proposed that, potentially, every level of processing shared by target and flankers (i.e., feature level and letter level) provides a source of target-flanker interaction, with the higher level processes either producing additional interference or, conversely, facilitating target recognition through the operation of top-down constraints reducing interference arising at the feature level.

To investigate the role of feature interactions and higher level factors in LM, in Experiments 1-6, target letter recognition was assessed under various flanker conditions. As baselines, both isolated letters and letters flanked by other letters were presented. Experiments 1 and 2 showed that target recognition accuracy was reduced, relative to both baselines when the flanking letters were rotated $180^{\circ}$ in the picture plane. A post hoc analysis showed that this effect was not due to facilitated processing of flanker-target-flanker strings that produced a meaningful word. Experiment 3 showed that rotating the flanking letters did not affect target recognition when targets were also rotated. This argues that flanker rotation per se does not impair target recognition. To sustain the argument that LM between upright targets and rotated flankers, as observed in Experiments 1 and 2, is solely attributable to interactions among visual features, one would have to assume that rotated and upright flankers differ in terms of visual features. Experiment 4, by using rotated and upright pseudoletter flankers, showed that this assumption is not tenable. Rather, the results of Experiment 4 support the idea that the rotation of the 
flankers does not alter feature processing. The stronger masking effect with rotated relative to upright letter flankers in Experiments 1 and 2 must therefore be attributed to some higher level processing. Of course, rotated flankers are harder to recognize than upright flankers, which might be responsible for the impaired target recognition with rotated flankers. This account was examined in Experiment 5, by presenting letter-like pseudoletters as flankers - that is, flankers that were even harder to recognize than rotated flankers. The results confirmed that these pseudoletter flankers impaired target recognition accuracy. In contrast, in Experiment 6, nonletter stimuli were presented as flankers that are also coded at a higher level, (i.e., digits). Digit flankers enhanced target letter recognition rather than impairing it. Taken together with the results of the previous experiments, effects of higher level processing on LM were clearly demonstrated.

LM effects are usually characterized by a statistical interaction between the factors of retinal eccentricity and spacing (Bouma, 1970; Loomis, 1978; Wolford \& Chambers, 1983), which were systematically varied in all experiments. In each experiment, an interaction between target eccentricity and spacing and main effects of the two variables were obtained, replicating earlier studies (Bouma, 1970; R. J. Jacobs, 1979).

The flanker effects exhibited systematic interactions with these variables: Whenever there was a significant main effect of flanker type (Experiments 1,2,5, and 6), there were also two-way and three-way interactions of flanker type with eccentricity and with spacing or with both. Importantly, the eccentricity and spacing variables only modified the magnitude of the flanker effect, they never changed its direction. In contrast, whenever there was no main effect of flanker type (Experiments 3 and 4), there were no interactions involving this variable. This pattern of results argues that eccentricity and spacing determine the level of recognition accuracy that can be attained, but not the strength of interference between target and the various types of flanker. For the purpose of the present study, it seemed sufficient to demonstrate that LM was influenced by higher level attributes of the flankers and that this influence was evident at all target eccentricity and target-flanker spacing conditions. Nevertheless, it may be interesting in future research to further specify the role of target eccentricity and spacing, by adjusting stimulus duration so as to permit a constant level of recognition performance under all eccentricity and spacing conditions, while avoiding the existence of ceiling or floor effects. Any special effects of spacing and eccentricity ought to become manifest under these conditions.

If the eccentricity and spacing effects were due to feature interactions, with flankers that differ in feature composition from upright letter flankers, one would expect target recognition accuracy to be affected in a different way, relative to letter flankers, by increasing eccentricity and decreasing spacing. However, the effects of the various types of flanker were manifest under all target eccentricity and spacing conditions. Moreover, in all experiments, the effects of flanker type were most marked with medium setting of the eccentricity and spacing variables, in which case recognition performance was in the range of $40 \%-60 \%$. Thus, feature interactions alone cannot account for the present data.

As mentioned above, one assumption was that all levels of processing that target and flankers have in common provide a potential source of interference. Thus, stronger interaction effects were expected for targets flanked by letters than for targets flanked by nonletters, because nonletters and letters should interact only during featurelevel processing, but not during letter coding. Clearly, such an account cannot be maintained.

An alternative explanation might work along the following lines. Higher level letter codes organize the information derived by feature coding processes via top-down links. In analogy to word recognition, where a word superiority effect is observed, there is also a letter superiority effect (see also Reingold \& Joliceur, 1993). Word superiority means that a letter presented in a word context is recognized better than a letter in a nonword context (Reicher, 1969; Wheeler, 1970). Hierarchical models explain these effects by assuming facilitatory top-down processing (McClelland \& Rumelhart, 1981), such that word recognition does not require the recognition of all individual letters, in particular when partial evidence is sufficient to specify the word. Moreover, word-level activation enhances letter recognition, so that even letters that were only partially identified by the lower level processes become reportable.

Applied to letter recognition, a similar mechanism can account for the effects obtained in our experiments: In the same way as word superiority means that letters are better recognized in words than in nonwords, letter superiority would mean that features are better recognized in letters than in nonletters. This is supported by our findings: Pseudoletter flankers, which can interact with the target letters only at a featural level, produced more interference than letter flankers, which can interact at both the feature level and the letter level. Therefore, with letter flankers, the interaction between visual features must have been reduced through derivation of the flankers' letter-level codes. In analogy to the assumptions for letter and word recognition, one can assume that similar mechanisms underlie feature and letter processing: One of the initial stages in letter recognition is the activation of feature nodes, which in turn activate letter nodes. As many letter nodes become activated in parallel, their activation feeds back to feature coding, organizing information at this level and reducing feature interactions. When activation achieved at the letter level is reduced, as with pseudoletter flankers, low-level feature interactions are suppressed to a lesser extent. Abstract coding occurs not only through activation of units in a mental alphabet representation but also through activation of other abstract representations, such as representations for digits. As a result, nonletter flankers facilitate target recognition whenever they have access to an abstract representation, and they impair target recognition performance whenever they do not.

Nevertheless, even when higher level codes are activated, LM can still be observed. This suggests that, al- 
though letters are processed, the perfect organization of information at the feature level may not be achieved. If this is the case, then processing at the next level, where word codes are derived, might reduce LM effects even further. In support of this, LM is hardly detectable when letters are processed as a word (Massaro \& Cohen, 1994; McClelland \& Rumelhart, 1981). This is the very meaning of word superiority. By implication, LM is an effect obtained whenever a letter string is not perceived as a word. Further investigations of LM in words might help us arrive at a complete understanding of the phenomenon.

The effects of target eccentricity and spacing on LM have led to the hypothesis that LM arises from interference at an early visual level of processing (Bouma, 1970; Wolford, 1975). However, our data argue that the term lateral masking leads to a misconception concerning the cognitive processes involved in the effect. The results reported here show a clear dependence of LM on higher level coding. Whenever a flanker gains access to a higher level code, the interaction effects produced by the flanker are modulated by this, and recognition of the target letter is facilitated.

\section{REFERENCES}

Aubert, H., \& Foerster, R. (1857). Beiträge zur Kenntniss des indirekten Sehens. Untersuchungen über den Raumsinn der Retina [Contributions to the knowledge of indirect vision. Studies on the spatial sense of the retina]. Archiv für Ophthalmologie, 3(2), 1-37.

BerINGER, J. (1993). ERTS: Experimental Run Time System, 3.00. Frankfurt.

Bouma, H. (1970). Interaction effects in parafoveal letter recognition. Nature, 226, 177-178.

Bouma, H. (1978). Visual search and reading: Eye movements and functional visual field. In J. Requin (Ed.), Attention and performance VII (pp. 631-644). Hillsdale, NJ: Erlbaum.

CoOper, L. A., \& Shepard, R. N. (1973). Chronometric studies of the rotation of mental images. In W. G. Chase (Ed.), Visual information processing (pp. 75-176). New York: Academic Press.

DunCAN, J., \& Humphreys, G. (1992). Beyond the search surface: Visual search and attentional engagement. Journal of Experimental $P_{s y-}$ chology: Human Perception \& Performance, 18, 578-588.

EGETH, H. E., \& SANTEE, J.L. (1981). Conceptual and perceptual components in interletter inhibition. Journal of Experimental Psychology: Human Perception \& Performance, 7, 506-517.

ESTES, W. K. (1972). Interactions of signal and background variables in visual processing. Perception \& Psychophysics, 12, 278-286.

EsTES, W. K. (1982). Similarity-related channel interactions in visual processing. Journal of Experimental Psychology: Human Perception \& Performance, 8, 353-382.

Estes, W. K., Allmeyer, D. H., \& Reder, S. M. (1976). Serial position functions for letter identification at brief and extended exposure durations. Perception \& Psychophysics, 19, 1-15

GreenberG, S. N., \& Krueger, L. E. (1983). Effect of letter orientation and sequential redundancy on the speed of letter search. Memory \& Cognition, 11, 181-191.

Heller, D., Huckauf, A., \& Nazir, T. A. (1995). Interaktionseffekte im seitlichen Gesichtsfeld: Eine Erweiterung des Ansatzes von Bouma (1970) [Interaction effects in peripheral vision: An extension of Bouma's approach (1970)]. Zeitschrift für Experimentelle und Angewandte Psychologie, 42, 386-418.

JaCOBS, A. M., NAZIR, T. A., \& Heller, O. (1989). Perception of lowercase letters in peripheral vision: A discrimination matrix based on saccade latencies. Perception \& Psychophysics, 46, 95-102.

JACOBS, R. J. (1979). Visual resolution and contour interaction in the fovea and periphery. Vision Research, 19, 1187-1195.
KEHRER, L. (1985). Über Klassifikationsleistungen beim visuellen Suchen: Untersuchungen zur Funktionsgrundlage des Kategorieneffektes [Classification in visual search: Studies on the functional foundation of the category effect]. Unpublished doctoral dissertation, University of Bielefeld, Germany.

KRUEGER, L. E. (1984). The category effect in visual search depends on physical rather than conceptual differences. Perception \& Psychophysics, 35, 558-564.

LoOMIS, J. M. (1978). Lateral masking in foveal and eccentric vision Vision Research, 18, 335-338.

MASON, M. (1982). Recognition time for letters and nonletters: Effects of serial position, array size, and processing order. Journal of Experimental Psychology: Human Perception \& Performance, 8, 724-738.

Massaro, D. W., \& COHEN, M. M. (1994). Visual, orthographic, and lexical influences in reading. Journal of Experimental Psychology. Human Perception \& Performance, 20, 1107-1128.

Massaro, D. W., \& KlitZKe, D. (1979). The role of lateral masking and orthographic structure in letter and word recognition. Acta Psychologica, 43, 413-426.

McClelland, J. L., \& Rumelhart, D. E. (1981). An interactive activation model of context effects in letter perception: Part 1. An account of basic findings. Psychological Review, 88, 375-407.

Mewhort, D. J. K., Marchetti, F. M., \& Campbell, A. J. (1982). Blank characters in tachistoscopic recognition: Space has both a symbolic and a sensory role. Canadian Journal of Psychology, 36, 559-574.

MiLler, J. (1991). The flanker compatibility effect as a function of visual angle, attentional focus, visual transients, and perceptual load: A search for boundary conditions. Perception \& Psychophysics, 49, 270-288.

PaAp, K. R., Newsome, S. L., McDonald, J. E., \& Schvanefeldt, R. W. (1982). An activation-verification model for letter and word recognition: The word superiority effect. Psychological Review, 89, 573-594.

Prinzmetal, W. (1992). The word-superiority effect does not require a T-scope. Perception \& Psychophysics, 51, 473-484.

ReICHER, G. M. (1969). Perceptual recognition as a function of meaningfulness of stimulus material. Journal of Experimental Psychology, 81, 725-280

Reingold, E. M., \& Joliceur, P. (1993). Perceptual versus postperceptual mediation of visual context effects: Evidence from the lettersuperiority effect. Perception \& Psychophysics, 53, 166-178.

SANOCKI, T. (1987). Visual knowledge underlying letter perception: Font-specific, schematic tuning. Journal of Experimental Psychology: Human Perception \& Performance, 13, 267-278.

SANOCK1, T. (1988). Font regularity constraints on the process of letter recognition. Journal of Experimental Psychology: Human Perception \& Performance, 14, 472-480.

SHAPIRO, R. G. \& KRUEGER, L. E. (1983). Effect of surround on targetletter processing. Journal of Experimental Psychology: Human Perception \& Performance, 9, 547-559.

Strasburger, H., Harvey, L. O., JR., \& Rentschler, I. (1991). Contrast thresholds for identification of numeric characters in direct and eccentric view. Perception \& Psychophysics, 49, 495-508.

STYLES, E. A., \& AllporT, D. A. (1986). Perceptual integration of identity, location and colour. Psychological Research, 48, 189-200.

Teichner, W. H., \& Krebs, M. J. (1974). Laws of visual choice reaction time. Psychological Review, 81, 75-98.

TOWNSEND, J. T., TAYLOR, S. G., \& BROWN, D. R. (1971). Lateral masking for letters with unlimited viewing time. Perception \& Psychophysics, 10, 375-378.

VAN DER HEIJDEN, A. H. C. (1992). Selective attention in vision. London: Routledge.

WhEELER, D. D. (1970). Processes in word recognition. Cognitive Psychology, 1, 59-85.

WOLFORD, G. (1975). Perturbation model for letter identification. Psychological Review, 82, 184-199.

Wolford, G., \& Chambers, L. (1983). Lateral masking as a function of spacing. Perception \& Psychophysics, 33, 129-138.

(Manuscript received January 29, 1997; revision accepted for publication January 15, 1998.) 